\title{
BREAST IMAGING USING 3D ELECTRICAL IMPEDENCE TOMOGRAPHY
}

\author{
Sachin Prasad N*, Dana Houserkova, Jan Campbell
}

\author{
Department of Radiology*, Faculty of Medicine and Dentistry, Palacky University, Olomouc, Czech Republic \\ e-mail:drsachinprasadn@yahoo.co.in
}

Received: March 21, 2008; Accepted (with revisions): June 8, 2008

Key words: Mammography/Ultrasonography/Bio-impedance/3D EIT/Breast Imaging

Aim: To determine the diagnostic efficiency of 3D Eletrical Impedance Tomography (EIT) compared to Mammography (MG) and Ultrasonography (USG) in imaging the breast.

Materials and Methods: A group of 88 patients presenting with various breast complaints was examined using combined Mammography and Ultrasonography (MG \& USG) or either of these modalities alone. The same patients were then examined using the 3D EIT imaging system "MEIK".

The findings were then compared. The sensitivity of these modalities for this group of patients were later determined and statistically analysed.

Results: Of the total of 88 patients, 59 findings were "suspicious" by any of the 3 modalities alone or by their combination. EIT had a sensitivity of $77.8 \%$ compared to MG with a sensitivity of $83.3 \%$ and USG with a sensitivity of $94.4 \%$ regarding cases of fibrocystic mastitis. For cases involving cysts, EIT had $100 \%$ sensitivity which was the same as that for USG compared to MG with a sensitivity of only $81 \%$. Among cases of fibroadenoma, EIT had a sensitivity of just $68.8 \%$ compared to MG with a sensitivity of $87.5 \%$ and USG with a sensitivity of $75 \%$. Finally among cases of carcinoma, EIT had a sensitivity of $75 \%$ compared to the sensitivity of $100 \%$ of MG and USG in our group of patients. The study revealed that there was no overall significant difference in sensitivity between MG-USG $(p=0.219)$ and MG-EIT ( $p=0.779$ ) and USG-EIT ( $p=0.169)$. However, in regard to identifying cysts there was significant difference in the sensitivity of MG compared to USG \& EIT suggesting that EIT has a role in these cases.

Conclusion: Electrical impedance could be used as an adjunct to Mammography and Ultrasonography for breast cancer detection. However, the differentiation of malignant from benign lesions based on impedance measurements needs further investigation. Multifrequency electrical impedance imaging appears the most promising for detecting breast malignancies but methodological improvements need to be made to realise its potential.

\section{INTRODUCTION}

Breast cancer is the second most prevalent cancer in females worldwide. The primary risk factors that have been identified are sex, age, childbearing, hormones, a high-fat diet, alcohol intake, obesity, and environmental factors such as tobacco use and radiation. Breast cancer, like other forms of cancer, is considered to be the final outcome of multiple environmental and hereditary factors and therefore the best way to prevent mortality is early detection. In the past 15 years, mortality has been reduced significantly in part due to screening using film-screen mammography. Mammography (MG) screening is thus currently considered the best method available for mass screening in the early detection of breast cancer ${ }^{1}$. Various other modalities are also used as adjuncts to mammography for the differentiation of potentially suspicious breast lesions. Of these, Ultrasonography over the years has been and is still a valuable non-invasive adjunct to mammography. Electrical impedance tomography (EIT) is also a potential, noninvasive diagnostic technique for imaging the human breast. The electrical impedance scanning device it uses does not emit any radiation and consists of a hand-held scanning probe and a computer screen that displays two-dimensional images of the breast. An electrode is placed on the patient's arm. A very small amount of electric current, about the same amount used by a small penlight battery, is transmitted through the electrode and into the body. The current travels through the breast, where it is measured by the scanning probe placed over the breast. An image is generated from the measurements of electrical impedance. Breast cancer cells conduct electricity better than normal breast cells and tend to have lower electrical impedance and breast tumors can appear as bright white spots on the computer screen. Different types of tissue have different electrical impedance levels (electrical impedance is a measurement of how fast electricity travels through a given material). Some types of tissue have high electrical impedance while others have low electrical impedance. Breast tissue that is cancerous has a much lower electrical impedance (conducts electricity much better) than normal breast tissue. Electrical impedance scanning devices are being tried along with conventional mammography to detect breast cancer. The primary goal of this study was to evaluate the clinical efficiency of EIT in comparison to Mammography and Ultrasonography in detecting various lesions of the breast. 


\section{MATERIALS AND METHODS}

A group of 88 patients with various complaints of the breast were examined using MG \& USG or either of these modalities alone as required. The patients were then also assessed using EIT after verbal consent. A sensitivity test was observed to compare results and conclude on the 3 modalities. The new modality study to monitor its efficiency over MG\& USG was clearly explained. The EIT findings were then compared with the findings from MG \& USG. The subjective opinion of the patient regarding the time, stress and physical discomfort of EIT in comparison to MG \& USG was also given importance in this study. MG was performed using (Diamond, Instrumentarium Imaging, Tuusula, Finland),USG was done using lineararray transducer with a center frequency of $8-11 \mathrm{MHz}$ was performed using Logiq $500 \mathrm{MD}$, General Electric, Solingen, Germany. EIT was done using the 3D EIT imaging system MEIK, consisting of 256 electrodes, electrical current at $0,5 \mathrm{MA} / 50 \mathrm{kHz}$ (FIG 1) which permits 3D images of conductivity distribution in breast tissue up to $4.6 \mathrm{~cm}$ and more below the skin surface. The sensitivity of these modalities in this group of patients were later determined and statistically correlated.

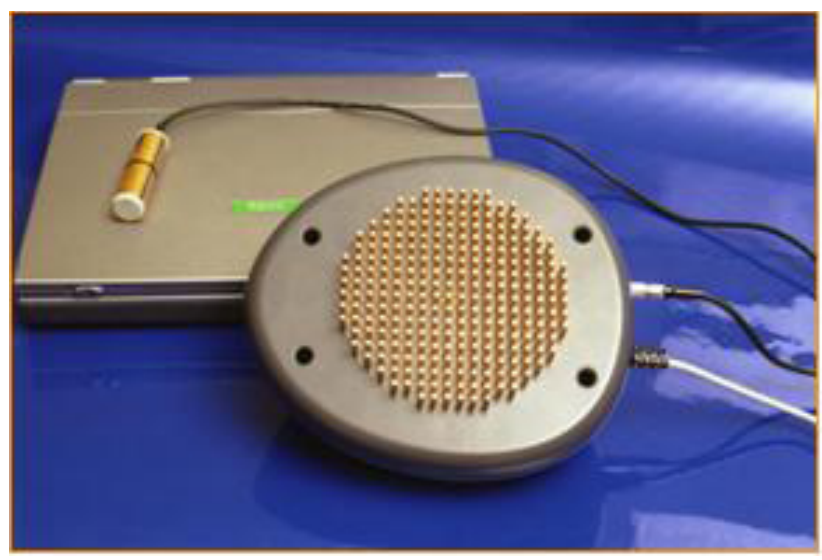

Fig. 1. (MEIK). The Electrical impedance tomography unit which was used for examining the patients.

\section{RESULTS}

Of a total of 88 patients who reported various complaints of the breast, 59 were "suspect" from either EIT, MG or USG or in some patients by all 3 modalities. The histopathological reports also revealed the abnormalities. These showed that of a total of 18 cases of fibrocystic mastitis, EIT was successful in detecting 14 with a sensitivity of $77.8 \%$ compared to 15 detected by MG with a sensitiv-

Table 1. HPE findings comparative analysis.

\begin{tabular}{|c|c|c|c|c|}
\hline \multirow[b]{2}{*}{ HPE } & \multirow[b]{2}{*}{ No: of Pts } & \multicolumn{3}{|c|}{ Sensitivity ( 95\% Confidence level) } \\
\hline & & Mammography & USG & EIT \\
\hline Fibrocystic mastitis & 18 & $\begin{array}{l}(15 / 18), 83.3 \% \\
(58.6 \%-96.4 \%)\end{array}$ & $\begin{array}{l}(17 / 18), 94.4 \% \\
(72.7 \%-99.9 \%)\end{array}$ & $\begin{array}{c}(14 / 18), 77.8 \% \\
(52.4 \%-93.4 \%)\end{array}$ \\
\hline Cyst & 21 & $\begin{array}{l}(17 / 21), 81.0 \% \\
(58.1 \%-94.6 \%)\end{array}$ & $\begin{array}{l}(21 / 21), 100 \% \\
(83.9 \%-100 \%)\end{array}$ & $\begin{array}{l}(21 / 21), 100 \% \\
(83.9 \%-100 \%)\end{array}$ \\
\hline Fibroadenoma & 16 & $\begin{array}{l}(14 / 16), 87.5 \% \\
(61.7 \%-98.5 \%)\end{array}$ & $\begin{array}{l}(12 / 16), 75.0 \% \\
(57.2 \%-92.7 \%)\end{array}$ & $\begin{array}{l}(11 / 16), 68.8 \% \\
(41.3 \%-88.9 \%)\end{array}$ \\
\hline Carcinoma & 4 & $\begin{array}{c}(4 / 4), 100 \% \\
(39.8 \%-100 \%)\end{array}$ & $\begin{array}{c}(4 / 4), 100 \% \\
(39.8 \%-100 \%)\end{array}$ & $\begin{array}{c}(3 / 4), 75.0 \% \\
(19.4 \%-99.4 \%)\end{array}$ \\
\hline Total & 59 & $\begin{array}{l}(50 / 59), 84.7 \% \\
(77.1 \%-92.3 \%)\end{array}$ & $\begin{array}{l}(54 / 59), 91.5 \% \\
(85.6 \%-97.4 \%)\end{array}$ & $\begin{array}{c}(49 / 59), 83.1 \% \\
(75.1 \%-91.0 \%)\end{array}$ \\
\hline
\end{tabular}

Table 2. p-value.

\begin{tabular}{|l|c|c|c|}
\hline Compare sensitivity & $\begin{array}{c}\text { MG vs. USG } \\
\text { p-value }\end{array}$ & $\begin{array}{c}\text { MG vs. EIT } \\
\text { p-value }\end{array}$ & $\begin{array}{c}\text { USG vs. EIT } \\
\text { p-value }\end{array}$ \\
\hline Fibrocystic mastitis & 0.298 & 0.697 & 0.163 \\
\hline Cyst & 0.042 & 0.042 & 1.000 \\
\hline Fibroadenoma & 0.372 & 0.197 & 0.708 \\
\hline Carcinoma & 1.000 & 0.326 & 0.326 \\
\hline
\end{tabular}



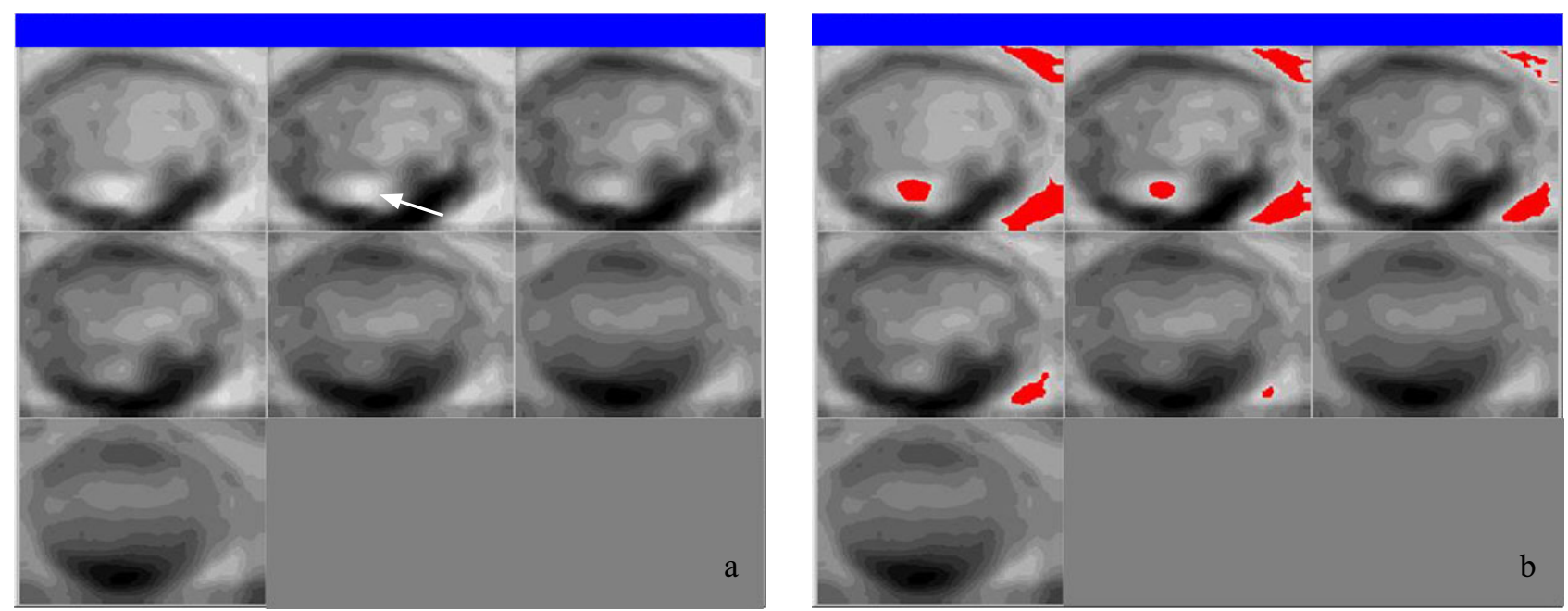

Fig. 2a-b. The black arrow shows a clearly defined hyper-impedance area (6 o'clock) of a roundish shape can be observed on the 2 nd scanning plane which is suggestive of breast cancer. Fig $2 \mathrm{~b}$ is a tomogram with a highlighted area in red in 6 o'clock position with electrical conductivity $>0.95$ conventional units.
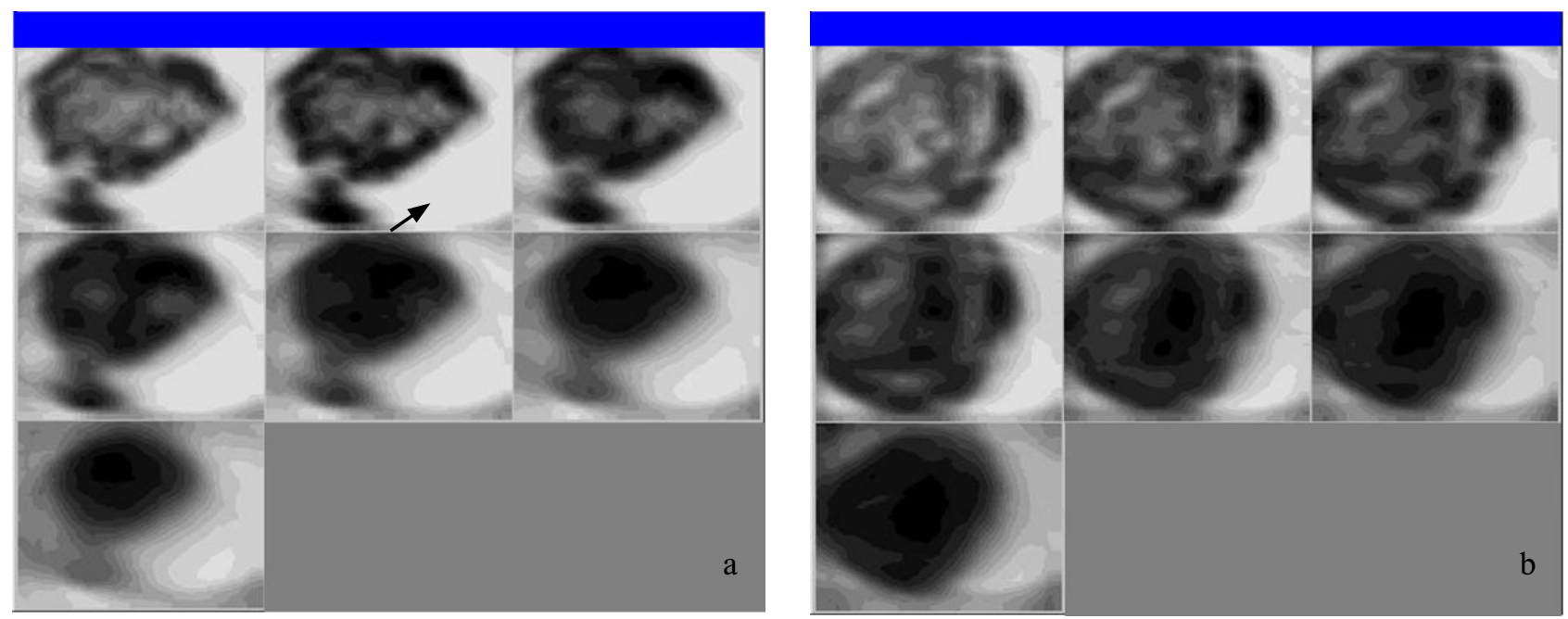

Fig. 3a-b. Electrical impedance tomogram of a 72-year-old patient with an extensive breast cancer of the right mammary gland. It indicates a considerable decrease of the contacting surface of the affected gland. Due to low vascularization of the malignant tumour hyper-impedance areas on the tomogram can't be defined. There is a sharp reaction of the subcutaneous tissue around the space-occupying lesion in form of a hyper-impedance outline marked by the black arrow. Fig $3 \mathrm{~b}$ shows a tomogram of the part of healthy gland in the same picient.

ity of $83.3 \%$ and 17 detected by USG with a sensitivity of $94.4 \%$. Of 21 cases of cyst, EIT detected all 21 with $100 \%$ sensitivity the same as that for USG compared to only 17 of MG with a sensitivity of only $81 \%$. Of a total of 16 cases of fibroadenoma, EIT detected only 11 with a sensitivity of just $68.8 \%$ compared to 14 detected by MG with a sensitivity of $87.5 \%$ and 12 detected by that of USG with a sensitivity of $75 \%$. Finally, among 4 cases of carcinoma EIT detected only 3 with a sensitivity of $75 \%$ compared to the sensitivity of $100 \%$ for MG and USG in this group of patients. Table 1 below gives a clear picture of the HPE (Histopathological entities) comparative analysis.
From this study there was no overall statistical significant difference in sensitivity between MG and USG $(p=0.219)$ or MG-EIT $(p=0.779)$ or USG-EIT $(p=0.169)$. However, with regard to identifying cysts there was significant difference in the sensitivity of MG compared to USG \& EIT. This was because as indicated in Table 1, only 17 of 21 were detected as having a cyst by MG whereas all 21 were detected by USG \& EIT. This is shown clearly in Table 2. (Figs $2 \& 3$ show the images of some breast conditions under MEIK.) 


\section{DISCUSSION}

Different imaging systems (e.g. electrical, magnetic, and ultrasound) rely on a wide variety of physical properties, and the datasets obtained from such systems provide only partial information about the unknown true state. Experimental studies with EIT have shown that significant changes occur in the electrical properties of breast cancer tissue compared to the surrounding normal tissue $e^{1-10}$. This phenomenon motivated studies on cancer detection using electrical impedance techniques. Some evidence has been found that malignant breast tumours have lower electrical impedance than surrounding normal tissues ${ }^{18}$. This observation has led to the proposal that electrical impedance could be used as an indicator for breast cancer detection. However, the separation of malignant tumours from benign lesions based on impedance measurements needs further investigation ${ }^{3,4,7-15}$ as also found this study that the differentiaton of malignant and benign lesions was poor and showed no particular characteristic impedance. An assessment of technologies for breast cancer screening and diagnosis conducted by the Institute of Medicine of the National Academy of Sciences (2001) concluded that clinical data suggest the technology [EIT] could play a role in breast cancer detection, but more study is needed to define its role in relation to existing technologies $^{11}$. Stojadinovic et al. (2005) presented preliminary results on the use of EIT for the early detection of breast cancer in young women ${ }^{1}$. They stated that EIT appears promising for early detection of breast cancer, and identification of young women at increased risk for having the disease at the time of screening. Positive EIT-associated breast cancer risk compares favorably with relative risks of conditions commonly used to justify early breast cancer screening ${ }^{7-9}$. The authors also noted that more data are needed to ascertain more accurately the actual sensitivity. These investigators also believe that EIT has promise as a breast cancer screening modality for a group of women for whom no effective screening modality currently exists. The sensitivity found in this study was in accordance to the above authors views of EIT. EIT seems to identify a population at increased risk for having breast cancer for whom further imaging examinations may be warranted.

\section{CONCLUSION}

Progress in the development of EIT breast imaging system will definitely help to promote other systems and applications based on the EIT and similar visualization methods. Electrical impedance could be used as an ad- junct to Mammography and ultrasonography for breast cancer detection. However, the separation of malignant tumours from benign lesions based on impedance measurements needs further investigation as well. Multifrequency electrical impedance imaging appears promising for detecting breast malignancies, but improvements must be made before the method reaches its full potential.

\section{REFERENCES}

1. Martin G, Martin R, Brieva MJ, Santamaria L. Electrical impedance scanning in breast cancer imaging: Correlation with mammographic and histologic diagnosis. Eur Radiol. 2002; 12(6):1471-1478.

2. Stojadinovic A, Nissan A, Gallimidi Z, et al. Electrical impedance scanning for the early detection of breast cancer in young women: Preliminary results of a multicenter prospective clinical trial. J Clin Oncol. 2005; 23(12):2703-2715.

3. Cherepenin V, Karpov A, Korjenevsky A, et al. A 3D electrical impedance tomography (EIT) system for breast cancer detection. Physiol Meas. 2001; 22(1):9-18.

4. Cherepenin VA, Karpov AY, Korjenevsky AV, et al. Three-dimensional EIT imaging of breast tissues: System design and clinical testing. IEEE Trans Med Imaging. 2002; 21(6):662-667.

5. Malich A, Fuchsjager M. Electrical impedance scanning in classifying suspicious breast lesions. Invest Radiol. 2003; 38(5):302-304.

6. S I Fields, M Rossman and E Phillips, Adjunctive improvement of mammographic accuracy using electrical impedance scanning (EIS). Radiology 209 (1998), pp. 272-273.

7. Athanasiou A, Vanel D, Fournier L, Balleyguier C. Optical mammography: A new technique for visualizing breast lesions in women presenting non palpable BIRADS 4-5 imaging findings: Preliminary results with radiologic-pathologic correlation. Cancer Imaging. 2007; 7:34-40.

8. Taulbee P. FDA panel rejects Mirabel's bioimpedance device for breast scanning. FDC Reports: The Gray Sheet. 2006; 32(36):8.

9. Wersebe A, Siegmann K, Krainick U, et al. Diagnostic potential of targeted electrical impedance scanning in classifying suspicious breast lesions. Invest Radiol. 2002; 37(2):65-72.

10. Zou Y, Guo Z. A review of electrical impedance techniques for breast cancer detection. Med Eng Phys. 2003; 25(2):79-90.

11. National Academy of Sciences, Institute of Medicine, National Cancer Policy Board, Committee on the Early Detection of Breast Cancer. Mammography and Beyond: Developing Technologies for the Early Detection of Breast Cancer. Washington, DC: National Academy Press; 2001.

12. Kerner TE, Paulsen KD, Hartov A, et al. Electrical impedance spectroscopy of the breast: Clinical imaging results in 26 subjects. IEEE Trans Med Imaging. 2002; 21(6):638-645.

13. Kerner TE, Hartov A, Soho SK, et al. Imaging the breast with EIS: an initial study of exam consistency. Physiol Meas. 2002; 23(1):221-236.

14. Ohmine Y, Morimoto T, Kinouchi Y, et al. Noninvasive measurement of the electrical bioimpedance of breast tumors. Anticancer Res. 2000; 20(3B):1941-1946.

15. Malich A, Boehm T, Facius M, et al. Differentiation of mammographically suspicious lesions: Evaluation of breast ultrasound, MRI mammography and electrical impedance scanning as adjunctive technologies in breast cancer detection. Clin Radiol. 2001; 56(4):278-283. 